NBER WORKING PAPER SERIES

\title{
ELECTORAL COMPETITION WITH FAKE NEWS
}

Gene M. Grossman

Elhanan Helpman

Working Paper 26409

http://www.nber.org/papers/w26409

\author{
NATIONAL BUREAU OF ECONOMIC RESEARCH \\ 1050 Massachusetts Avenue \\ Cambridge, MA 02138 \\ October 2019
}

We thank Alberto Alesina and Guido Tabellini for helpful discussions and Benjamin Niswonger for research assistance. Grossman thanks CREI for its hospitality. The views expressed herein are those of the authors and do not necessarily reflect the views of the National Bureau of Economic Research.

NBER working papers are circulated for discussion and comment purposes. They have not been peer-reviewed or been subject to the review by the NBER Board of Directors that accompanies official NBER publications.

(C) 2019 by Gene M. Grossman and Elhanan Helpman. All rights reserved. Short sections of text, not to exceed two paragraphs, may be quoted without explicit permission provided that full credit, including $\left({ }^{\circ}\right.$ notice, is given to the source. 
Electoral Competition with Fake News

Gene M. Grossman and Elhanan Helpman

NBER Working Paper No. 26409

October 2019

JEL No. D72,D78

\section{ABSTRACT}

Misinformation pervades political competition. We introduce opportunities for political candidates and their media supporters to spread fake news about the policy environment and perhaps about parties' positions into a familiar model of electoral competition. In the baseline model with full information, the parties' positions converge to those that maximize aggregate welfare. When parties can broadcast fake news to audiences that disproportionately include their partisans, policy divergence and suboptimal outcomes can result. We study a sequence of models that impose progressively tighter constraints on false reporting and characterize situations that lead to divergence and a polarized electorate.

Gene M. Grossman

International Economics Section

Department of Economics

Princeton University

Princeton, NJ 08544

and NBER

grossman@princeton.edu

Elhanan Helpman

Department of Economics

Harvard University

1875 Cambridge Street

Cambridge, MA 02138

and NBER

ehelpman@harvard.edu 


\section{Introduction}

Misinformation on the part of the public makes for bad lawmaking on the part of the government.

Keohane (2010)

Do Facts Matter?, Hochschild and Einstein (2015) ask in the title of their monograph on the role of misinformation in American politics that opens with the above quote. Do unwise policies result when politicians and the media spread false information and the citizenry accepts it unquestioningly before deciding how to vote? The authors use case studies to argue that "people's willingness to use mistaken factual claims in their voting and public engagement is ... dangerous to a democratic polity." (2015, p.14)

Here we address a similar question with game-theoretic tools. We take the well-known probabilistic voting model of Lindbeck and Weibull (1987) as our baseline environment. In this setting, there are two political parties that differ in exogenous ways and a population of voters with heterogenous partisanships. The parties compete by staking positions on a "pliable" policy issue that voters consider along with their idiosyncratic assessments of the parties' fundamentals. In a setting with accurately informed voters, the election delivers pliable policies that maximize aggregate welfare. But what if voters are ill informed and parties can compete by making false claims about the policy environment and the positions supported by themselves and their rivals? Under what circumstances will the potential for spreading "fake news" distort the parties' positions away from those that are socially desirable? Will the parties make competing claims that polarize the electorate or will they broadcast similar announcements? We ask these questions in a sequence of models with increasingly tight constraints on the scope for false reporting. First, we give the parties free rein to make claims both about a parameter that affects the desirability of alternative policies (i.e., the "state of the world") and about their and their rival's positions on the matter. The parties reach different audiences and have a greater chance of being heard by their own partisans. Next, we restrict the parties to announce their own position accurately, while still allowing false claims about the state of the world and about the rival's intentions. Finally, we suppose that voters know both parties' positions, but still may be misled about the attractiveness of alternative policy options. In each case, we ask whether the parties converge or diverge in their positions and announcements and whether the fake news distorts the ultimate policy outcome.

While misinformation has long been a tool in political competition, recent trends have heightened concern about the spread of misleading or "fake" news. Social media and other internet outlets enable politicians and their allies to reach ever-larger, targeted audiences. Guess et al. (2018) estimate that one in four Americans visited a fake news website in the six weeks before the 2016 U.S. presidential election - where fake news is defined as the most extreme form of misleading information inasmuch as its content is intentionally and verifiably false. Allcott and Gentzkow (2017) document the increasing role that social media play as a source of political information and argue that "people who get news from Facebook (or other social media) are less likely to receive 
evidence about the true state of the world that would counter an ideologically aligned but false story" (p.221). Moreover, these authors and Silverman and Singer-Vine (2016) report survey evidence that many voters have difficulty distinguishing real and fake news and that many believe the false claims they encounter. Guess et al. (2018) provide evidence of selective exposure to fake news sources: Republican voters were more likely to receive news from pro-Trump sources than Democratic voters. This characteristic of the information technology features prominently in our modeling of the parties' strategic use of fake news.

We contribute to a small literature on the role of imperfect information in electoral competition. An early contribution by Baron (1994) features informed and uninformed voters, with the latter responding mechanically to campaign spending financed by interest groups. Glaeser et al. (2005) explain "extremism" in policy positions in a model in which individuals vote only if the expected benefit exceeds an idiosyncratic voting cost and potential voters are more likely to learn the position of their affiliated party than the position of the nonaffiliated party. In this paper, we study formally the strategic use of misinformation in political competition.

\section{A Model of Electoral Competition with Fake News}

Two political parties, $L$ and $R$, vie for electoral support. The parties differ in some exogenouslygiven ways that appeal differently to the heterogeneous voters. Voters also care about a "pliable" policy that will be contested in the election. The parties use their positions and broadcasts on this issue instrumentally to woo voters. Individuals may have access to disparate information and hold different beliefs about the intentions of the two parties with regard to the pliable policy and about the state of the policy environment.

Specifically, consider a voter $i$ who believes that party $L$ would carry out the pliable policy $m_{i}^{L}$, that party $R$ would carry out the pliable policy $m_{i}^{R}$, and that the state of the world is characterized by the parameter $\theta_{i}$, a scalar that impacts her assessment of the alternative policy choices. This individual votes for party $L$ if and only if

$$
u\left(m_{i}^{L}, \theta_{i}\right)-u\left(m_{i}^{R}, \theta_{i}\right) \geq \eta_{i}
$$

where $\eta_{i}$ reflects her relative preference for party $R$ based on the fundamental differences between the parties. In the unit mass of voters, the preference parameter $\eta_{i}$ is drawn from a well-behaved cumulative distribution function, $F(\eta)$.

The utility function $u(m, \theta)$ is increasing in $\theta$, concave in $m$, twice continuously differentiable, and supermodular. Moreover, the value of $m$ that maximizes $u(m, \theta)$ is finite for all $\theta$ in the feasible range, $[\underline{\theta}, \bar{\theta}]$. It follows that the value of $m$ that maximizes $u(m, \theta)$ is increasing in $\theta$ and lies in the bounded range $[\underline{m}, \bar{m}]$, where $\underline{m} \equiv \arg \max _{m} u(m, \underline{\theta})$ and $\bar{m} \equiv \arg \max _{m}(m, \bar{\theta})$. We assume that $\theta_{i} \in[\underline{\theta}, \bar{\theta}], m_{i}^{L} \in[\underline{m}, \bar{m}]$ and $m_{i}^{R} \in[\underline{m}, \bar{m}]$ for all $i$; i.e., voters believe that $\theta$ falls within the feasible range and that each party's position corresponds to one that is optimal for some feasible

state. We also assume that for any feasible combination of $\theta_{i}, m_{i}^{L}$, and $m_{i}^{R}$, there exist values of 
$\eta_{i}$ in the support of $F(\eta)$ such that $i$ prefers $R$ and values of $\eta_{i}$ such that $i$ prefers $L$.

Voters access news from disparate sources. A fraction $\lambda^{I}$ of the populace receives accurate information. These voters know the true value of $\theta$, which is $\theta^{I} \in(\underline{\theta}, \bar{\theta})$, and the true positions and intentions of the parties, $m^{L}$ and $m^{R}$. The remaining voters form their impressions based on reports from biased sources. These sources may include partisan media outlets or announcements (e.g., "tweets") made directly by the politicians themselves. The sources assert the state of the policy environment and possibly the positions of one or both of the parties. These assertions might bear no relationship to the truth. We refer to such misinformation broadly as "fake news."

Voters choose their news source non-strategically, but their listening habits reflect their ideological proclivities. In particular, a voter who prefers party $R$ on ideological grounds and who does not have access to reliable information is more likely to follow a media source that is partial to party $R$ than is another voter who fundamentally prefers party $L$. Letting $\pi(\eta)$ denote the probability that a voter with $\eta_{i}=\eta$ who accesses fake news hears the reports of a source that is aligned with party $R$, we assume $\pi^{\prime}(\eta)>0$. The uninformed voters take what they hear at face value; if their partisan information source reports, for example, that $\theta=\tilde{\theta}$, then they use this value in assessing the (perceived) policy positions. Such unquestioning acceptance of biased news is an extreme assumption, but the studies we cited in the introduction provide evidence that fake news has powerful effects on followers' beliefs and the extreme assumption allows us to capture this reality in a simple way.

It is straightforward to construct examples that fit our framework based on recent policy controversies. For example, the policy $m$ might represent the number of immigrants that are admitted into a country, while $\theta$ affects (inversely) the social and economic cost of absorbing immigrants. Then $u_{m \theta}>0$ applies if the optimal number of immigrants increases as the cost of absorption falls (see appendix). News outlets might exaggerate the cost of immigration in one direction or the other, while perhaps also misrepresenting the parties' positions on the matter. Or the policy $m$ might represent the size of a tariff on imports, while $\theta$ represents (inversely) the induced foreign price. The optimal tariff rate is greater when exporters "pay for the tariff"; i.e., when the induced foreign price is low. In this case, the media might exaggerate the pass-through of tariffs to domestic prices and possibly the parties' openness to trade.

\subsection{The Parties' Objectives and Actions}

Each party seeks to maximize its share of the aggregate vote. Party $J$ (or its partisan media outlet) reports the state of the policy environment as $\theta^{J}$, with free rein to announce any $\theta^{J} \in[\underline{\theta}, \bar{\theta}]$. The party also reports its own position to be $m^{J J}$ and that of its rival to be $m^{J \widetilde{J}}$, where $\widetilde{J}$ denotes the party that is not $J$. The parties might feel constrained in these latter announcements, either because voters have ready access to accurate information of this sort or because the parties perceive a reputational cost from misrepresenting their positions. In order to understand how such constraints affect the prospects for divergent positions and suboptimal policies, we proceed to analyze three cases with increasingly tighter reporting constraints. First, we allow party $J$ to 
claim any position $m^{J J} \in[\underline{m}, \bar{m}]$ as its own and any $m^{J \widetilde{J}} \in[\underline{m}, \bar{m}]$ as its rival's intention. Next, we suppose that each party must report its own position accurately, but can misrepresent that of its rival. Finally, we constrain all reports of policy positions to be truthful.

In each case, we can compute the parties' vote shares as a function of the announcements. The votes for party $L$ comprise those among the fully-informed that prefer $m^{L}$ to $m^{R}$ with the knowledge that $\theta=\theta^{I}$, those among the uninformed voters that obtain their news from sources favoring party $L$ and that prefer $m^{L L}$ to $m^{L R}$ under the (possibly mistaken) belief that $\theta=\theta^{L}$, and those among the uninformed voters that obtain their news from sources favoring party $R$ and prefer $m^{R L}$ to $m^{R R}$ under the belief that $\theta=\theta^{R}$. Summing these components, we have

$$
\begin{aligned}
s^{L} & =\lambda^{I} F\left[u\left(m^{L}, \theta^{I}\right)-u\left(m^{R}, \theta^{I}\right)\right] \\
& +\left(1-\lambda^{I}\right) \int_{-\infty}^{u\left(m^{L L}, \theta^{L}\right)-u\left(m^{L R}, \theta^{L}\right)}[1-\pi(\eta)] d F(\eta) \\
& +\left(1-\lambda^{I}\right) \int_{-\infty}^{u\left(m^{R L}, \theta^{R}\right)-u\left(m^{R R}, \theta^{R}\right)} \pi(\eta) d F(\eta)
\end{aligned}
$$

where $s^{L}$ is the vote share for party $L$ and $s^{R}=1-s^{L}$ is the remaining vote share. ${ }^{1}$ Here, the first term on the right-hand side of (1) gives the fraction of the $\lambda^{I}$ knowledgeable voters that prefer $m^{L}$ to $m^{R}$ in light of the baseline preferences for the two parties, the second term gives the fraction of the $1-\lambda^{I}$ uninformed voters that, with probability $1-\pi(\eta)$, obtain their reports from an $L$-leaning source and decide to vote for party $L$ in view of their induced belief that $m^{L}=m^{L L}$, $m^{R}=m^{L R}$ and $\theta=\theta^{L}$, and the third term gives the fraction of the $1-\lambda^{I}$ uninformed voters that, with probability $\pi(\eta)$, obtain their news from an $R$-leaning source and decide nonetheless to vote for party $L$ after being told that $m^{L}=m^{R L}, m^{R}=m^{R R}$ and $\theta=\theta^{R}$.

\subsection{The Full-Information Benchmark}

As a benchmark, we review the outcome of electoral competition with complete and accurate information; see, for example, Lindbeck and Weibull (1987). The full-information benchmark is captured in our model by the special case with $\lambda^{I}=1$. In this case, the policy $m^{J}$ that maximizes $s^{J}$ given $m^{\widetilde{J}}$ is $m^{I}=\arg \max _{m} u\left(m, \theta^{I}\right)$; therefore, the positions converge to the policy that maximizes the representative voter's welfare given the true state of the world.

\subsection{Reports about the Policy Environment}

In what follows, we will always assume that the parties or their media representatives report about the state of the policy environment in the final stage of the political game. The incentives to report about $\theta$ at this stage are common to the settings with and without fake news about policy positions.

\footnotetext{
${ }^{1}$ Note that $s^{L}$ is deterministic in this setting with a continuum of voters. As is well known, it would be straightforward to add a valence shock reflecting the uncertain popularity of each party at a moment in time in order to make $s^{L}$ random and thus leave the parties with a real electoral contest.
} 
We consider these incentives now.

To this end, suppose that the parties have staked the positions $m^{L}$ and $m^{R}$, and that the audience for the broadcasts by party $J$ believe these positions to be $m^{J J}$ and $m^{J \widetilde{J}}$ for $J=L, R$. Given these beliefs, the announcement about $\theta$ by party $J$ can only affect voting by those that follow the $J$-leaning source. Party $J$ maximizes its vote share by claiming that $\theta=\theta^{J}$, where

$$
\theta^{J}=\arg \max _{\theta \in[\underline{\theta}, \bar{\theta}]} u\left(m^{J J}, \theta\right)-u\left(m^{J \widetilde{J}}, \theta\right), J=L, R
$$

The supermodularity of $u(\cdot)$ then implies

$$
\theta^{J}=\left\{\begin{array}{ll}
\bar{\theta} & \text { for } m^{J J}>m^{J \widetilde{J}} \\
\underline{\theta} & \text { for } m^{J J}<m^{J \widetilde{J}}
\end{array} \quad, J=L, R .\right.
$$

Evidently, each party issues extreme pronouncements on the state of the world. If the audience for party $J$ 's broadcasts believes that party $J$ will invoke a policy greater than will its rival, the party wants its listeners to believe that high values of $m$ are maximally beneficial to their utility. If the audience believes the opposite to be true about the ordering of the party's positions, then it wants its listeners to believe that low values of $m$ are best.

In the following sections, we consider the equilibrium choices of policies in settings with increasingly tighter constraints on reporting. We assume throughout that the parties first choose their positions (either simultaneously or sequentially) in an initial stage of electoral competition and later broadcast their claims about the policy environment and positions.

\section{Unconstrained Reporting of Policy Positions}

In this section, we suppose that parties (or their media allies) can report whatever they like about their own position and that of their rival to the audiences for their respective broadcasts. Those that hear the news reported by party $R$ will vote based on a comparison of $u\left(m^{R R}, \theta^{R}\right)$ and $u\left(m^{R L}, \theta^{R}\right)$. Clearly, this comparison is independent of the position actually taken by party $L$ and the news broadcast by that party. Similarly, those that receive their news from party $L$ compare $u\left(m^{L L}, \theta^{L}\right)$ to $u\left(m^{L R}, \theta^{L}\right)$, which is independent of the position and announcements of party $R$. Evidently, the outcome is the same whether the parties stake their positions simultaneously or sequentially.

When broadcasting its news, party $J$ seeks to make itself look maximally appealing to those that hear its claims, while also trying to make its rival look maximally repugnant. With this objective in mind, party $J$ faces a choice between two extreme alternatives. It might announce its own policy position to be the one most preferred when the state of the world is as high as possible while claiming that the rival intends a policy at the opposite end of the spectrum, or it might announce its own policy position to be that most preferred when the state of the world is as low as possible while claiming that its rival supports the policy at the upper end of the spectrum. Among these 
alternatives, it chooses the one that creates the greatest gap in audience perceptions. Note that both parties face the same incentives in this regard: if $u(\bar{m}, \bar{\theta})-u(\underline{m}, \bar{\theta})>u(\underline{m}, \underline{\theta})-u(\bar{m}, \underline{\theta})$, then each maximizes the perception gap by claiming the highest possible value of $\theta$ along with its own alleged support for the policy that goes along with that state and the intentions of the rival to enact the lowest credible policy level; otherwise they each choose the opposite extreme announcements. Thus

$$
\left(\theta^{J}, m^{J J}, m^{J \widetilde{J}}\right)=\left\{\begin{array}{ll}
(\bar{\theta}, \bar{m}, \underline{m}) & \text { for } u(\bar{m}, \bar{\theta})-u(\underline{m}, \bar{\theta})>u(\underline{m}, \underline{\theta})-u(\bar{m}, \underline{\theta}) \\
(\underline{\theta}, \underline{m}, \bar{m}) & \text { for } u(\bar{m}, \bar{\theta})-u(\underline{m}, \bar{\theta})<u(\underline{m}, \underline{\theta})-u(\bar{m}, \underline{\theta})
\end{array}, \text { for } J=L, R .\right.
$$

What positions do the parties adopt in the initial stage(s) of the electoral game? In the current setting, these choices affect only the behavior of knowledgeable voters. Party $L$ chooses $m^{L}$ to maximize its appeal among these voters, i.e., to maximize $u\left(m^{L}, \theta^{I}\right)-u\left(m^{R}, \theta^{I}\right)$. It has a dominant strategy to choose $m^{I}$. Party $R$ perceives the same dominant strategy. In short, both parties converge on the pliable policy that is socially optimal, much as in the setting with complete and accurate information.

We summarize in

Proposition 1 Suppose that the parties choose their actual positions simultaneously or sequentially and that each party is subsequently unconstrained in its reports about the state of the world, its own policy position, and that of its rival. Then the policy positions converge to those that maximize utility given the actual state of the world $\left(m^{L}=m^{R}=m^{I}\right)$ and the announcements converge to whichever extreme offers the greatest perception gap; either $\left(\theta^{L}, m^{L L}, m^{L R}\right)=\left(\theta^{R}, m^{R R}, m^{R L}\right)=(\bar{\theta}, \bar{m}, \underline{m})$ or $\left(\theta^{L}, m^{L L}, m^{L R}\right)=\left(\theta^{R}, m^{R R}, m^{R L}\right)=(\underline{\theta}, \underline{m}, \bar{m})$.

\section{Accurate Reporting of Own Positions}

Now suppose that the parties feel compelled to report accurately about their own intentions, but take leeway in misrepresenting the position of their rivals. As we have observed in Section 2.3, the parties should expect that reports about the state of the world will be extreme. If $m^{J \tilde{J}}<m^{J}$, party $J$ will announce that $\theta=\bar{\theta}$, whereas if $m^{J \tilde{J}}>m^{J}$, it will announce that $\theta=\underline{\theta}$. Anticipating such an announcement, party $J$ no longer wishes to choose the utility-maximizing policy position, $m^{I}$. On the one hand, such a position would capture the greatest number of votes among the knowledgeable voters, who recognize its optimality. On the other hand, such a position would not appeal to those who are (mis)led to believe that $\theta$ is extreme. When choosing its position, each party trades off the marginal appeal to knowledgeable voters against the marginal attraction to the audience for its fake news.

In order to conserve on space and focus on the roles of the probability function $\pi(\eta)$, we henceforth assume that $\eta$ is drawn from a uniform distribution on support $\left[\eta_{\min }, \eta_{\max }\right]$. With this assumption, the outcome in this section is the same no matter whether the platforms are chosen simultaneously or sequentially. 
To identify the equilibrium choices by the parties, we must first consider what false report each will subsequently make about the other's position. Suppose party $J$ has adopted a position of $m^{J}$. If $m^{J}$ is close to $\bar{m}$, party $J$ makes itself maximally attractive to its audience by reporting $\theta=\bar{\theta}$ and $m^{J \widetilde{J}}=\underline{m}$. Alternatively, if $m^{J}$ is close to $\underline{m}$ the party makes itself maximally attractive by reporting $\theta=\underline{\theta}$ and $m^{J \widetilde{J}}=\bar{m}$. In general, there is an intermediate value of $m$, say $\hat{m}$, such that if $m^{J}>\hat{m}$, party $J$ prefers to announce $\theta^{J}=\bar{\theta}$ and $m^{J \widetilde{J}}=\underline{m}$ to the alternative of announcing $\theta^{J}=\underline{\theta}$ and $m^{J \widetilde{J}}=\bar{m}$, whereas if $m^{J}<\hat{m}$, the opposite is true. It follows that $\hat{m}$ is defined by

$$
u(\hat{m}, \bar{\theta})-u(\underline{m}, \bar{\theta})=u(\hat{m}, \underline{\theta})-u(\bar{m}, \underline{\theta}) .
$$

Notice that the fake news reported by the two parties might converge or diverge. If both parties have adopted positions on the same side of $\hat{m}$, both will issue the same biased report about the policy environment and a similar (false) report about their rival's stance. Alternatively, if the two parties adopt positions on opposite sides of $\hat{m}$, then the parties will issue opposite extreme reports about the state of the world and correspondingly opposite reports about their rival's intentions.

We turn now to the choice of policy positions by the two parties. A party's choice of position affects voting by informed voters and by those that receive their information from its aligned media outlets. Therefore, each party has a dominant strategy that is independent of the policy choice of its rival and of the anticipated reports from media sources aligned with that rival. The dominant strategy maximizes aggregate votes among the two groups that are affected; for party $L$, $m^{L}=\arg \max _{m} V^{L}(m)$, where

$$
V^{L}(m)=\lambda^{I} u\left(m, \theta^{I}\right)+\left(1-\lambda^{I}\right)\left\{\begin{array}{ll}
\int_{\eta_{\min }}^{u(m, \underline{\theta})-u(\bar{m}, \underline{\theta})}[1-\pi(\eta)] d \eta & \text { for } m \leq \hat{m} \\
\int_{\eta_{\min }}^{u(m, \bar{\theta})-u(\underline{m}, \bar{\theta})}[1-\pi(\eta)] d \eta \quad \text { for } m \geq \hat{m}
\end{array},\right.
$$

whereas for party $R, m^{R}=\arg \max _{m} V^{R}(m)$, where

$$
V^{R}(m)=\lambda^{I} u\left(m, \theta^{I}\right)-\left(1-\lambda^{I}\right)\left\{\begin{array}{ll}
\int_{\eta_{\min }}^{u(\underline{\bar{m}}, \underline{\theta})-u(m, \underline{\theta})} \pi(\eta) d \eta & \text { for } m \leq \hat{m} \\
\int_{\eta_{\min }}^{u(\underline{m}, \bar{\theta})-u(m, \bar{\theta})} \pi(\eta) d \eta & \text { for } m \geq \hat{m}
\end{array} .\right.
$$

Notice that the vote counts depend on whether $m \geq \hat{m}$ or $m \leq \hat{m}$, because the subsequent reports by the affiliated media hinge on this distinction.

The policy choice for party $J$ lies strictly between $\underline{m}$ and $\bar{m}$, because the votes among the misinformed change negligibly when, for example, $m^{J}$ is increased slightly from $\underline{m}$, inasmuch as $u_{m}(\underline{m}, \underline{\theta})=0$. Meanwhile, such a small increase in $m^{J}$ above $\underline{m}$ would gain party $J$ a non-negligible share of votes among the fully informed. By a similar argument, $m^{J}<\bar{m}$ for $J=L, R$. Also, it is never optimal for party $J$ to choose $m^{J}=\hat{m}$. For such a position to be optimal, it would have to be the case that a small increase in $m^{J}$ from $\hat{m}$ loses vote in view of the subsequent reports of $\theta^{J}=\bar{\theta}$, while a small decrease in $m^{J}$ from $\hat{m}$ also loses vote with a subsequent report of $\theta^{J}=\underline{\theta}$. But, from (8) and (9), we see, for example, that $\lim _{m \searrow \hat{m}} V_{m}^{J}(m)>\lim _{m} \hat{m}_{m} V_{m}^{J}(m)$, because 
the supermodularity of $u(m, \theta)$ implies that $u_{m}(m, \bar{\theta})>u_{m}(m, \underline{\theta})$. Thus, the former cannot be negative when the latter is positive. It follows that $m^{J} \in(\underline{m}, \hat{m})$ or $m^{J} \in(\hat{m}, \bar{m})$, depending on which strategy yields more votes. In either case, a first-order condition holds as an equality for the chosen position; i.e., $V_{m}^{J}\left(m^{J}\right)=0$ for $J=L, R$.

Now suppose that the probability function $\pi(\eta)$ is symmetric, in the sense that $\pi(\eta)=1-\pi(-\eta)$ for all $\eta$; that is, the fraction of voters who favor party $R$ by an amount $\eta$ that receive their news from an $R$-leaning source is the same as the fraction of voters who favor $L$ by that same amount that obtain their news from an $L$-leaning source. Then, the parties face a similar trade-off between appealing to their audience and appealing to knowledgeable voters. That is, the $m^{L}$ that maximizes $V^{L}(m)$ in (8) is the same as the $m^{R}$ that maximizes $V^{R}(m)$ in (9). In this symmetric case, the policy positions converge, albeit not to the policy $m^{I}$ that maximizes aggregate welfare.

But suppose instead that $\pi(\eta) \neq 1-\pi(-\eta)$ for all $\eta \in\left[\eta_{\min }, \eta_{\max }\right]$. In such circumstances, there can be no equilibrium with policy convergence. Convergence would require the existence of a policy position, $\tilde{m}$, such that $V_{m}^{L}(\tilde{m})=V_{m}^{R}(\tilde{m})=0$. But suppose, for example, that $\tilde{m}>\hat{m}$. Then, using (8) and (9), this would further require

$$
\{1-\pi[u(\tilde{m}, \underline{\theta})-u(\bar{m}, \underline{\theta})]\} u_{m}(\tilde{m}, \underline{\theta})=\pi[u(\bar{m}, \underline{\theta})-u(\tilde{m}, \underline{\theta})] u_{m}(\tilde{m}, \underline{\theta})
$$

Since $u_{m}(\tilde{m}, \underline{\theta})>0$, this equation can be satisfied only if $1-\pi[u(\tilde{m}, \underline{\theta})-u(\bar{m}, \underline{\theta})]=\pi[u(\bar{m}, \underline{\theta})-u(\tilde{m}, \underline{\theta})]$, which is not possible when $\pi(\eta) \neq 1-\pi(-\eta)$ for all $\eta \in\left[\eta_{\min }, \eta_{\max }\right]$. An analogous argument applies when $\tilde{m}<\hat{m}$. So, the parties' positions must diverge in these (asymmetric) circumstances.

We have thus established the following (see the appendix for more formal details).

Proposition 2 Suppose that $F(\eta)$ is uniform, that the parties choose their actual positions simultaneously or sequentially, and that each party is subsequently unconstrained in its reports about the state of the world and the position of its rival, but must report its own position accurately. Then the policy positions converge $\left(m^{L}=m^{R}\right)$ if $\pi(\eta)=1-\pi(-\eta)$ for all $\eta$ and diverge $\left(m^{L} \neq m^{R}\right)$ if $\pi(\eta) \neq 1-\pi(-\eta)$ for all $\eta$. The announcements about the state of the world and the rival party's policy position converge if $\min \left\{m^{L}, m^{R}\right\}>\hat{m}$ or $\max \left\{m^{L}, m^{R}\right\}<\hat{m}$ and diverge if $\max \left\{m^{L}, m^{R}\right\}>\hat{m}>\min \left\{m^{L}, m^{R}\right\}$.

When will the parties stake positions on opposite sides of $\hat{m}$, so that their extreme fake-news reports polarize the electorate? To address this question, we consider further the relationship between vote share and policy position.

Anticipating the subsequent reporting, party $L$ chooses its policy position $m^{L}$, to maximize its vote share $s^{L}\left(m^{L}, m^{R}\right)$, which is a function of the policy positions of both parties. Party $R$ chooses $m^{R}$ to minimize party $L$ 's vote share. It follows from the expression for $s_{L}$ in (1), the definitions of $V^{L}\left(m^{L}\right)$ and $V^{R}\left(m^{R}\right)$ in (8) and (9), and the properties of the uniform distribution, $F(\eta)=\left(\eta-\eta_{\min }\right) /\left(\eta_{\max }-\eta_{\min }\right)$, that $s^{L}\left(m^{L}, m^{R}\right)$ satisfies

$$
\lambda^{I} \eta_{\min }+\left(\eta_{\max }-\eta_{\min }\right) s^{L}\left(m^{L}, m^{R}\right) \equiv V^{L}\left(m^{L}\right)-V^{R}\left(m^{R}\right) .
$$



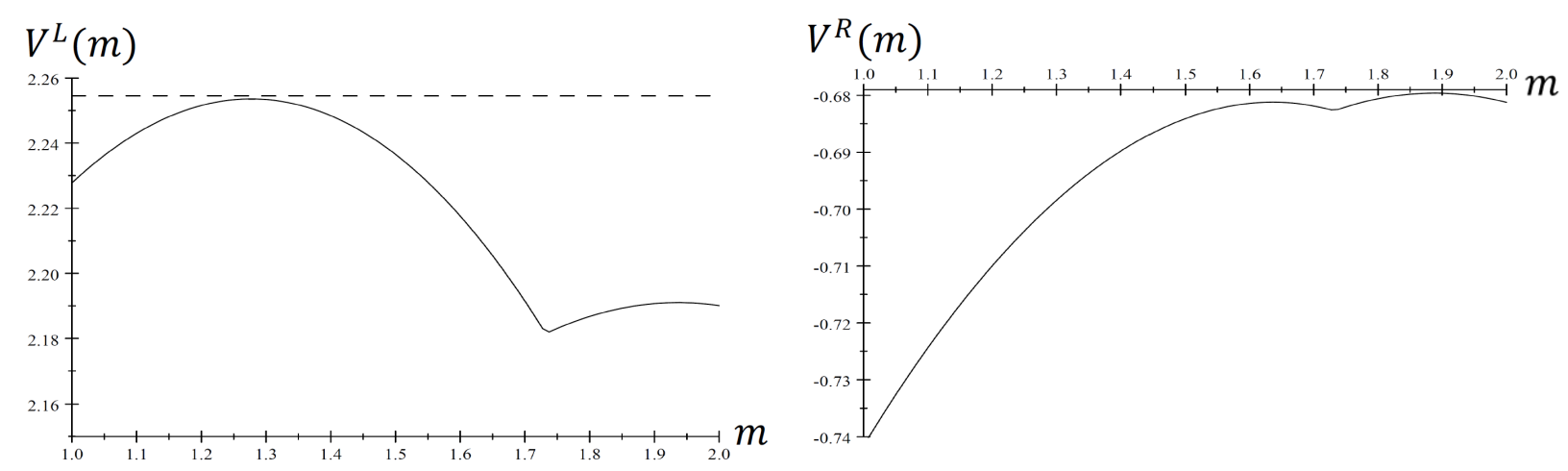

Figure 1: Accurate Reporting of Own Positions: Policy Divergence

The function $V^{L}(m)$ has two local maxima-one at $m_{-}^{L} \leq \hat{m}$ and another at $m_{+}^{L} \geq \hat{m}$ - and a local minimum at $m=\hat{m}$. The optimal policy for party $L$ is $m_{-}^{L}$ if $V^{L}\left(m_{-}^{L}\right)>V^{L}\left(m_{+}^{L}\right)$, and $m_{+}^{L}$ otherwise. Similarly, the function $V^{R}(m)$ has two local maxima, at $m_{+}^{R} \geq \hat{m}$ and at $m_{-}^{R} \leq \hat{m}$, and party $R$ chooses the one that yields the greater value of $V^{R}(m)$. As we have noted, each party's vote-maximizing strategy is independent of the choice by its rival.

The optimal strategy for each party clearly depends on the fraction of knowledgeable voters. When $\lambda^{I}$ is very small, for example, $u\left(m, \theta^{I}\right)$ plays little role in a party' choice of policy. Instead, the parties cater to their respective audiences by supporting policies close to $\underline{m}$ or close to $\bar{m}$, according to whether $u(\underline{m}, \underline{\theta})-u(\bar{m}, \underline{\theta})$ is larger or smaller than $u(\bar{m}, \bar{\theta})-u(\underline{m}, \bar{\theta})$. In this case, both parties choose nearly the same position, both on the same side of $\hat{m}$. Consequently, they both broadcast the same false news reports. ${ }^{2}$

At the other extreme, when $\lambda^{I}$ is close to one, the parties both cater mostly to the informed voters. In this case, both stake positions close to $m^{I}$. If $m^{I}>\hat{m}$, then each party $J$ reports $\theta^{J}=\bar{\theta}$ and $m^{J \widetilde{J}}=\underline{m}$, whereas if $m^{I}<\hat{m}$, each reports $\theta^{J}=\underline{\theta}$ and $m^{J \widetilde{J}}=\bar{m}$. Again, the fake news reports coincide and misinformed voters hold similar (incorrect) views, despite their disparately biased sources.

Although the parties' positions must fall on the same side of $\hat{m}$ when $\lambda^{I}$ is close to zero and when $\lambda^{I}$ is close to one, this need not be the case when the fraction of informed voters takes on an intermediate value. We illustrate an example of this in Figure 1. The figure depicts a case where $u(m, \theta)=m-m^{2} / 2 \theta$, with $\theta \in[1,2]$. We take $\pi(\eta)=e^{\eta} /\left(e^{\eta}+5\right)$, which implies $\pi(\eta)<1-\pi(-\eta)$ for all $\eta$ in the relevant range.

The figure shows $V^{L}(m)$ in the left panel and $V^{R}(m)$ in the right panel, for the case of $\lambda^{I}=0.4$ and $\theta^{I}=(\sqrt{3}+2) / 2 \approx 1.87$ (see the appendix for details). The dominant electoral strategy for party $L$ is $m^{L} \approx 1.3<\hat{m}$, while that for party $R$ is $m^{R} \approx 1.9>\hat{m}$. Then $L$ reports $\theta^{L}=1$ and $m^{L R}=2$, whereas $R$ reports $\theta^{R}=2$ and $m^{R L}=1$. In this example, the fact that $\pi(\eta)<1-\pi(-\eta)$ implies that party $R$ puts relatively more weight on the policy preferences of the informed voters compared to party $L$. For an intermediate value of $\lambda^{I}$ such as $\lambda^{I}=0.4$, party $L$ prefers to announce a policy below $\hat{m}$ and thus well below $m^{I}$, because its electoral strategy relies heavily on fake news.

\footnotetext{
${ }^{2}$ In the limit, when $\lambda^{I} \rightarrow 0$, the policy positions converge.
} 
Meanwhile, party $R$ that is less likely to reach uninformed voters relies on a strategy that caters to knowledgeable voters and chooses a position close to (but more extreme than) $m^{I} \approx 1.87$.

In the example, the reporting of fake news creates a polarized electorate; those that obtain their news from party $R$ come to believe that $\theta=\bar{\theta}=2$, while those that obtain their news from party $L$ believe instead that $\theta=\underline{\theta}=1$.

\section{Complete Information about Policy Positions}

In this section, we focus on a setting in which false reporting is confined to claims about the state of the policy environment. In this case, if the parties set their positions simultaneously, there does not exist any equilibrium in pure strategies; see the appendix for details. Inasmuch as the mixedstrategy equilibria are difficult to characterize, we limit our attention to the game in which parties move sequentially, with the incumbent party choosing its policy position before the challenger does so. After the positions are chosen and become common knowledge, the parties simultaneously report their fake news about the state of the world, $\theta$. Knowledgeable voters compare $u\left(m^{L}, \theta^{I}\right)$ to $u\left(m^{R}, \theta^{I}\right)$. Uninformed voters that access their news from a source partisan to party $J$ compare $u\left(m^{L}, \theta^{J}\right)$ to $u\left(m^{R}, \theta^{J}\right)$, for $J=L, R$.

We begin, as usual, with the final stage of the game. Given the chosen positions, $m^{L}$ and $m^{R}$, the parties issue reports about the state of the world. Each party wishes to render itself maximally attractive to its audience. By arguments that are familiar by now, party $J$ reports $\theta^{J}=\bar{\theta}$ if $m^{J}>m^{\widetilde{J}}$ and $\theta^{J}=\underline{\theta}$ if $m^{J}<m^{\widetilde{J}}$, for $J=L, R$.

Now consider the choice of position by party $L$, the challenger in this case. As before, the party trades off the appeal to knowledgeable voters of a policy close to $m^{I}$ versus the appeal to the misinformed voters of a policy closer to one of the extremes. The challenger might choose a policy above $m^{R}$, anticipating the ensuing fake-news reports of $\theta^{L}=\bar{\theta}$ and $\theta^{R}=\underline{\theta}$. Among these, the party's optimal choice is the one that maximizes

$$
\begin{aligned}
& s_{\text {above }}^{L}=\lambda^{I} F[u\left.\left(m, \theta^{I}\right)-u\left(m^{R}, \theta^{I}\right)\right]+ \\
&\left(1-\lambda^{I}\right)\left\{\int_{-\infty}^{u(m, \bar{\theta})-u\left(m^{R}, \bar{\theta}\right)}[1-\pi(\eta)] d F(\eta)+\int_{-\infty}^{u(m, \underline{\theta})-u\left(m^{R}, \underline{\theta}\right)} \pi(\eta) d F(\eta)\right\},
\end{aligned}
$$

which we denote by $m_{\text {above }}^{L}$. Alternatively, it might choose a policy below $m^{R}$, anticipating in this case that $\theta^{L}=\underline{\theta}$ and $\theta^{R}=\bar{\theta}$. The best choice among these is the one that maximizes

$$
\begin{aligned}
& s_{\text {below }}^{L}=\lambda^{I} F[u\left.\left(m, \theta^{I}\right)-u\left(m^{R}, \theta^{I}\right)\right]+ \\
&\left(1-\lambda^{I}\right)\left\{\int_{-\infty}^{u(m, \underline{\theta})-u\left(m^{R}, \underline{\theta}\right)}[1-\pi(\eta)] d F(\eta)+\int_{-\infty}^{u(m, \bar{\theta})-u\left(m^{R}, \bar{\theta}\right)} \pi(\eta) d F(\eta)\right\},
\end{aligned}
$$

which we denote by $m_{\text {below }}^{L}$. The party's best response to $m^{R}$ is the one that yields the greater vote 
share among these two options. We write the best response as $m^{L}\left(m^{R}\right)$ and the resulting vote share as $s^{L}\left(m^{R}\right)$.

In the first stage, the incumbent party $R$ chooses $m^{R}$, anticipating the reaction to its choice and recognizing that the electoral competition is a zero-sum game. Therefore, the incumbent maximizes its own vote share by setting

$$
m^{R} \in \underset{m \in[\underline{m}, \bar{m}]}{\arg \min } s^{L}(m) .
$$

To characterize the equilibrium outcomes, we observe first that the challenger party $L$ always can invoke a strategy of matching the policy position of the incumbent, which then ensures the party a fraction $F(0)$ of the votes. Clearly, no equilibrium outcome can give the challenger, party $L$, less than this share of the vote; i.e., $s^{L}(m) \geq F(0)$. It follows that party $R$ can do no better than the fraction $1-F(0)$ of the votes. If $R$ can find a position that induces $L$ to match, this option must be an equilibrium strategy for the incumbent.

We now argue that policy convergence never is an equilibrium outcome when $\pi(0)<1 / 2$; i.e., when an uninformed voter that is indifferent between the parties on ideological grounds is more likely to tune in to the broadcasts of the challenger than to those of the incumbent. To this end, we conjecture the existence of an equilibrium with $m^{R}=m^{L}=\tilde{m}$, and then show that, with $\pi(0)<1 / 2$, party $L$ can profitably deviate to win more than the share $F(0)$ of the votes.

If party $L$ deviates from matching $m^{R}$ and instead sets $m^{L}=m^{R}+\varepsilon, \varepsilon>0$, it will induce party $R$ to report $\theta^{R}=\underline{\theta}$, while its own subsequent report will be $\theta^{L}=\bar{\theta}$. The change in votes for a small $\varepsilon$ is

$$
d s_{+}^{L} / F^{\prime}(0)=\lambda^{I} u_{m}\left(\tilde{m}, \theta^{I}\right) \varepsilon+\left(1-\lambda^{I}\right)\left\{\pi(0) u_{m}(\tilde{m}, \underline{\theta})+[1-\pi(0)] u_{m}(\tilde{m}, \bar{\theta})\right\} \varepsilon .
$$

If, instead, the party deviates to $m^{L}=m^{R}-\varepsilon, \varepsilon>0$, it will induce party $R$ to report $\theta^{R}=\bar{\theta}$, while its own subsequent report will be $\theta^{L}=\underline{\theta}$. The vote change that results from this small deviation is

$$
d s_{-}^{L} / F^{\prime}(0)=-\lambda^{I} u_{m}\left(\tilde{m}, \theta^{I}\right) \varepsilon-\left(1-\lambda^{I}\right)\left\{\pi(0) u_{m}(\tilde{m}, \bar{\theta})+[1-\pi(0)] u_{m}(\tilde{m}, \underline{\theta})\right\} \varepsilon .
$$

Summing these two, we have

$$
\left(d s_{+}^{L}+d s_{-}^{L}\right) / F^{\prime}(0)=\left(1-\lambda^{I}\right)[1-2 \pi(0)]\left[u_{m}(\tilde{m}, \bar{\theta})-u_{m}(\tilde{m}, \underline{\theta})\right] \varepsilon
$$

But the supermodularity of $u(\cdot)$ implies $u_{m}(\tilde{m}, \bar{\theta})>u_{m}(\tilde{m}, \underline{\theta})$. Then, if $\pi(0)<1 / 2, d s_{+}^{L}+d s_{-}^{L}>0$, which implies that at least one of these deviations increases the vote share for party $L$. We have thus established

Proposition 3 Suppose that the parties choose their positions sequentially and these positions become known to all voters. Later, the parties simultaneously report the state of the world. If $\pi(0)<1 / 2$, the equilibrium policies diverge $\left(m^{L} \neq m^{R}\right)$ and there is polarization of fake news; if $m^{J}>m^{\widetilde{J}}, \theta^{J}=\bar{\theta}$ and $\theta^{\widetilde{J}}=\underline{\theta}$, for $J=L, R$. In the equilibrium, $s^{L}>F(0)$. 


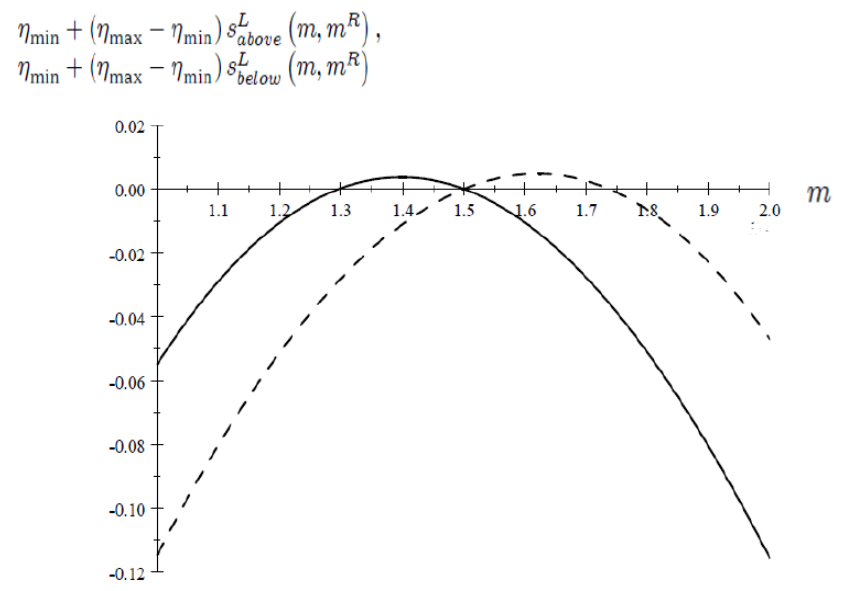

Figure 2: Complete Information about Policy Positions: Policy Convergence

Intuitively, when $\pi(0)<1 / 2$, the challenger can capitalize on the advantage it has in reaching the uninformed swing voters with fake news. Rather than mimic the proposal of the incumbent, it deviates in whichever direction yields the greater aggregate vote gain among the swing voters, once they have been induced by the fake news to believe one extreme or other about the state of the world.

In contrast, when $\pi(0)>1 / 2$, it is the incumbent that reaches a majority of the misinformed swing voters. Then the incumbent might be able to choose a position that leaves the challenger with no better option than to match. In Figure 2, we illustrate such an outcome. In this example, $u(m, \theta)=m-m^{2} / 2 \theta$ for $\theta \in[1,2]$ and $\pi(\eta)=e^{\eta} /\left(e^{\eta}+0.5\right)$. The solid

curve in the figure depicts $\eta_{\min }+\left(\eta_{\max }-\eta_{\min }\right) s_{\text {above }}^{L}\left(m, m^{R}\right)$, while the dashed curve depicts $\eta_{\min }+\left(\eta_{\max }-\eta_{\min }\right) s_{\text {below }}^{L}\left(m, m^{R}\right)$, both drawn for $m^{R}=1.5$. As the figure shows, $m^{L}=m^{R}$ represents the best response of party $L$ to $m^{R}=1.5$. Therefore, $m^{R}=1.5$ is an equilibrium action for party $R$ and the result is policy convergence, albeit not at the optimal policy, $m^{I} \simeq 1.87$.

\section{Conclusions}

We have introduced strategic misinformation into an otherwise standard model of electoral competition. As a benchmark, our model predicts policy convergence and welfare maximization when voters are fully informed. More generally, we assume that some voters have access to accurate information while others rely on biased sources to learn about the policy environment and perhaps the parties' policy positions. Among these uninformed voters, those that are partisan to some party are more likely to gain their information from a source that serves the interests of that party. We find circumstances in which fake news has real effects: the spread of such news may cause parties' policy positions to diverge and both may depart from the policy levels that are socially desirable. Such outcomes are most likely when each party or its media representative feels compelled to report accurately about its own position; then the parties face a trade-off in choosing their position between appealing to those who are well informed about the state of the world and those that will 
be misled to believe that the state is extreme in one direction or the other.

Our analysis is highly stylized and represents only a simple first step. Most importantly, the voters in our model are passive; they do not choose their information sources to achieve any particular objectives and they accept uncritically whatever it is that they hear. Further progress could perhaps be made by introducing some behavioral motives for voters' listening and reading habits and by allowing for some (limited) sophistication in their interpretation of the news. A more active role for the media would also be desirable, be they motivated by profits, partisanship, or career concerns. The salience of misinformation in modern day politics and our demonstration that fake news can matter for policy outcomes makes this a ripe topic for further research. 


\section{References}

[1] Allcott, Hunt, and Matthew Gentzkow. 2017. "Social Media and Fake News in 2016 Election." Journal of Economic Perspectives 31 (2): 211-6.

[2] Baron, David P. 1994. "Electoral Competition with Informed and Uninformed Voters." American Political Science Review 88 (1): 33-47.

[3] Glaeser, Edward L., Ponzetto, Giacomo A.M., and Jesse M. Shapiro. 2005. "Strategic Extremism: Why Republicans and Democrats Divide on Religious Values." The Quarterly Journal of Economics 120 (4): 1283-330.

[4] Guess, Andrew, Nyhan, Brendan, and Jason Reifler. 2018. "Selective Exposure to Misinformation: Evidence from the Consumption of Fake News during the 2016 U.S. Presidential Campaign." European Research Council Working Paper.

[5] Hochschild, Jennifer L., and Katherine Levine Einstein. 2015. Do Facts Matter: Information and Misinformation in American Politics. Norman, OK: University of Oklahoma Press.

[6] Keohane, Joe. 2010. "Imaginary Fiends: Crime in America Keeps Going Down. Why does the Public Refuse to Believe It?" The Boston Globe, February 14.

[7] Lindbeck, Assar, and Jörgen W. Weibull. 1987. "Balanced-budget Redistribution as the Outcome of Political Competition." Public Choice 52 (3): 273-97.

[8] Silverman, Craig, and Jeremy Singer-Vine. 2016. "Most Americans Who See Fake News Believe It, New Survey Says." BuzzFeed News, December 6. 


\section{Appendix}

In this appendix, we provide further details supporting the arguments made in the main text.

\section{Appendix for Section 2}

Recall that the utility function $u(m, \theta)$ is increasing in $\theta$, concave in $m$, twice continuously differentiable, and supermodular. The latter implies $u_{m \theta}(m, \theta)>0$. In Section 2 of the paper we mention an example in which $m$ is the number of immigrants and $\theta$ is inversely related to the cost of absorbing immigrants. The details of the example are as follows. Output is produced according to a constant-returns-to scale-technology, $f(n, m)=\left(n^{\alpha}+m^{\alpha}\right)^{1 / \alpha}, \alpha \in(0,1)$, where $n$ is the number of domestic workers, normalized so that $n=1$. Assuming that immigrants are paid a competitive wage, they generate surplus income for domestic residents of $b(m)=f(1, m)-f_{m}(1, m) m$. The function $b(m)$ is increasing and concave. Let $c(m) / \theta$ be the cost of absorbing $m$ immigrants, where $c(\cdot)$ is increasing and convex and $\theta$ is a cost shifter. Then $u(m, \theta) \equiv b(m)-c(m) / \theta$, with $u_{\theta}(m, \theta)>0$ and $u_{m \theta}(m, \theta)>0$. That is, $u(\cdot)$ is supermodular. The optimal number of immigrants, $m^{I}$, satisfies:

$$
u_{m}\left(m^{I}, \theta^{I}\right)=0
$$

It follows that the optimal number of immigrants is increasing in $\theta^{I}$.

\section{Appendix for Section 4}

In Section 4 of the paper, we discuss the case in which parties feel compelled to report accurately about their own intentions, but are free to misrepresent the position of their rivals. We elaborate on this case in this section of the appendix, assuming that $F(\eta)=\left(\eta-\eta_{\min }\right) /\left(\eta_{\max }-\eta_{\min }\right)$ is a uniform distribution on support $\left[\eta_{\min }, \eta_{\max }\right]$. As before, we assume that the support is broad enough that positive fractions of voters favor each party no matter what are the beliefs about the pliable policy environment and the parties' positions. In this case, equation (1) in the main text can be re-written as

$$
\begin{aligned}
\left(\eta_{\max }-\eta_{\min }\right) s^{L} & =\lambda^{I}\left[u\left(m^{L}, \theta^{I}\right)-u\left(m^{R}, \theta^{I}\right)-\eta_{\min }\right] \\
& +\left(1-\lambda^{I}\right)\left\{\int_{\eta_{\min }}^{u\left(m^{L}, \theta^{L}\right)-u\left(m^{L R}, \theta^{L}\right)}[1-\pi(\eta)] d \eta+\int_{\eta_{\min }}^{u\left(m^{R L}, \theta^{R}\right)-u\left(m^{R}, \theta^{R}\right)} \pi(\eta) d \eta\right\} .
\end{aligned}
$$

From equation (2) in the main text, which describes the optimal announcement of the policy environment, we have for this case

$$
\theta^{J}=\left\{\begin{array}{ll}
\bar{\theta} & \text { for } m^{J}>m^{J \widetilde{J}} \\
\underline{\theta} & \text { for } m^{J}<m^{J \widetilde{J}}
\end{array} \quad, J=L, R .\right.
$$


At the second stage, when the positions $m^{J}$ are given, party $L$ wishes to maximize $u\left(m^{L}, \theta^{L}\right)-$ $u\left(m^{L R}, \theta^{L}\right)$ while $R$ wishes to minimize $u\left(m^{R L}, \theta^{R}\right)-u\left(m^{R}, \theta^{R}\right)$. Therefore, the best response for party $J$ is to choose either $\theta^{J}=\bar{\theta}$ and $m^{J \widetilde{J}}=\underline{m}$, which is optimal if

$$
u\left(m^{J}, \bar{\theta}\right)-u(\underline{m}, \bar{\theta})>u\left(m^{J}, \underline{\theta}\right)-u(\bar{m}, \underline{\theta}),
$$

or else $\theta^{J}=\underline{\theta}$ and $m^{J \widetilde{J}}=\bar{m}$, which is optimal if the inequality runs in the opposite direction. Supermodularity of $u(m, \theta)$ implies that $u(m, \bar{\theta})-u(m, \underline{\theta})$ is increasing in $m$. In addition,

$$
\begin{aligned}
& u\left(m^{J}, \bar{\theta}\right)-u(\underline{m}, \bar{\theta})>u\left(m^{J}, \underline{\theta}\right)-u(\bar{m}, \underline{\theta}) \text { for } m^{J}=\bar{m}, \\
& u\left(m^{J}, \bar{\theta}\right)-u(\underline{m}, \bar{\theta})<u\left(m^{J}, \underline{\theta}\right)-u(\bar{m}, \underline{\theta}) \text { for } m^{J}=\underline{m} .
\end{aligned}
$$

Therefore there exists an $\hat{m} \in(\underline{m}, \bar{m})$ that satisfies

$$
u(\hat{m}, \bar{\theta})-u(\underline{m}, \bar{\theta})=u(\hat{m}, \underline{\theta})-u(\bar{m}, \underline{\theta}) .
$$

Accordingly, $\left\{\theta^{J}, m^{J \widetilde{J}}\right\}=\{\bar{\theta}, \underline{m}\}$ is the best strategy when $m^{J}>\hat{m}$ and $\left\{\theta^{J}, m^{J \widetilde{J}}\right\}=\{\underline{\theta}, \bar{m}\}$ is the best strategy when $m^{J}<\hat{m}$. For $m^{J}=\hat{m}$, party $J$ is indifferent between the two strategies. Note that party $J$ has a dominant strategy in the second stage of the game. This finding is summarized in

Lemma 1 For given choices of $m^{L}$ and $m^{R}$ in the first stage of the game and truthful reporting of own positions, party $J$ has a dominant strategy in the second stage of the game that is independent of its rival's play. This strategy is given by

$$
\left\{\theta^{J}, m^{J \widetilde{J}}\right\}=\left\{\begin{array}{ll}
\{\bar{\theta}, \underline{m}\} & \text { for } m^{J}>\hat{m}, \\
\{\underline{\theta}, \bar{m}\} & \text { for } m^{J}<\hat{m},
\end{array} \quad J=L, R\right.
$$

where $\hat{m}$ is implicitly defined in (6). For $m^{J}=\hat{m}$ party $J$ is indifferent between $\{\bar{\theta}, \underline{m}\}$ and $\{\underline{\theta}, \bar{m}\}$.

Proposition 4 It follows from this discussion that, for given values of $m^{J}, J=L, R$, the vote share of party L, satisfies

$$
\lambda^{I} \eta_{\min }+\left(\eta_{\max }-\eta_{\min }\right) s^{L}=V^{L}\left(m^{L}\right)-V^{R}\left(m^{R}\right)
$$

where $V^{L}(m)$ and $V^{R}(m)$ are defined as in equations (3) and (4) in the text. For convenience, we reproduce these definitions here:

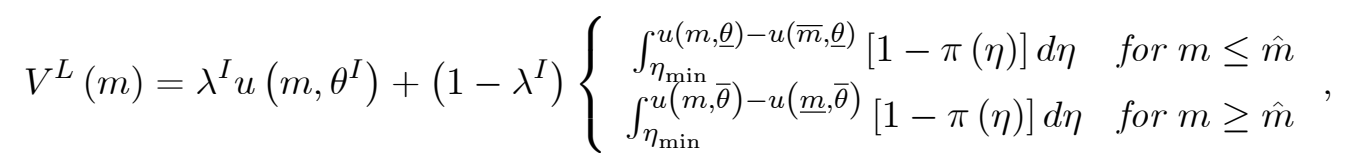




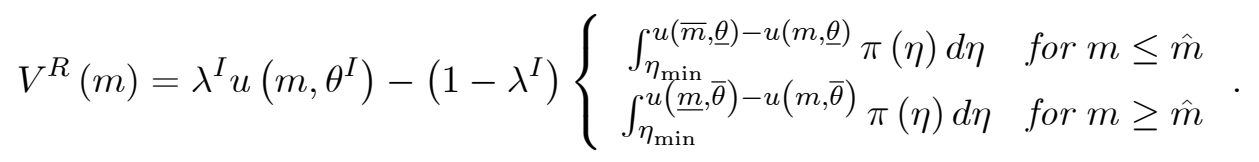

Now consider the first stage of the game. Party $L$ seeks to maximize $s^{L}$ while party $R$ seeks to minimize it. It follows from (7) that the best party $L$ can do is to choose an $m^{L}$ that maximizes $V^{L}(m)$. This solution does not depend on $m^{R}$. Similarly, party $R$ chooses an $m^{R}$ to maximizes $V^{R}(m)$ and the solution does not depend on $m^{L}$. It follows that each party has a dominant strategy in the first stage of the game and the same values for $m^{J}, J=L, R$, are equilibrium values in the simultaneous-move game and the sequential-move games. We have proven

Lemma 2 With truthful reporting of own positions, each party has a dominant strategy, $m^{J}, J=$ $L, R, m^{L}=\arg \max _{m \in[\underline{m}, \bar{m}]} V^{L}(m)$ and $m^{R}=\arg \max _{m \in[\underline{m}, \bar{m}]} V^{R}(m)$, where $V^{L}(m)$ is given in (8) and $V^{R}(m)$ is given in (9).

Next, note that the optimal strategy $m^{J}, J=L, R$, satisfies $\underline{m}<m^{J}<\bar{m}$, because the slope of $V^{J}(m)$ is positive at $\underline{m}$ and negative at $\bar{m}$. Also, $m^{J}=\hat{m}$ is not an optimal strategy. For $m^{L}=\hat{m}$ to be an optimal strategy the following inequalities would have to be satisfied:

$$
\lim _{m \nearrow \hat{m}} \frac{d V^{L}(m)}{d m} \geq 0 \geq \lim _{m \searrow \hat{m}} \frac{d V^{L}(m)}{d m} .
$$

Using (8), these inequalities hold if and only if

$$
u_{m}(m, \underline{\theta}) \geq 0 \geq u_{m}(m, \bar{\theta})
$$

which violates the supermodularity of $u(m, \theta)$. A similar argument establishes that $m^{R}=\hat{m}$ is not an optimal strategy for party $R$. It follows that $m^{J}$ is interior and either $m^{J} \in(\underline{m}, \hat{m})$ or $m^{J} \in(\hat{m}, \bar{m})$ for $J=L, R$.

Furthermore, if the probability function $\pi(\eta)$ is symmetric, in the sense that $\pi(\eta)=1-\pi(-\eta)$ for all $\eta \in\left[\eta_{\min }, \eta_{\max }\right]$, then $V^{L}(m)$ and $V^{R}(m)$ are maximized at the same value of $m$ (i.e., the peak on either side of $\hat{m}$ is attained for the same value of $m$ by both parties). In such circumstances, $m^{L}=m^{R}$ and there is policy convergence. We next show that if $\pi(\eta) \neq 1-\pi(-\eta)$ for all $\eta \in\left[\eta_{\min }, \eta_{\max }\right]$, then there can be no equilibrium with policy convergence. Namely, $m^{L} \neq m^{R}$. To prove this, first suppose that $m^{L}=m^{R}=\tilde{m}, \tilde{m} \in(\underline{m}, \hat{m})$. Then, it must be that

$$
\left.\frac{d V^{L}(m)}{d m}\right|_{m=\tilde{m}}=\left.\frac{d V^{R}(m)}{d m}\right|_{m=\tilde{m}}=0
$$

Using (8) and (9), this would imply

$$
\{1-\pi[u(\tilde{m}, \underline{\theta})-u(\bar{m}, \underline{\theta})]\} u_{m}(\tilde{m}, \underline{\theta})=\pi[u(\bar{m}, \underline{\theta})-u(\tilde{m}, \underline{\theta})] u_{m}(\tilde{m}, \underline{\theta})
$$

Since $u_{m}(\tilde{m}, \underline{\theta})<0$, this equation can be satisfied only if $1-\pi[u(\tilde{m}, \underline{\theta})-u(\bar{m}, \underline{\theta})]=\pi[u(\bar{m}, \underline{\theta})-u(\tilde{m}, \underline{\theta})]$, 
which is not possible when $\pi(\eta) \neq 1-\pi(-\eta)$ for all $\eta \in\left[\eta_{\min }, \eta_{\max }\right]$. A similar argument establishes that $m^{L}=m^{R}=\tilde{m}, \tilde{m} \in(\hat{m}, \bar{m})$, also cannot arise in any equilibrium. We therefore have

Lemma 3 With truthful reporting of own positions and $\pi(\eta) \neq 1-\pi(-\eta)$ for all $\eta \in\left[\eta_{\min }, \eta_{\max }\right]$, equilibrium policies do not converge, i.e., $m^{L} \neq m^{R}$. In contrast, if $\pi(\eta)=1-\pi(-\eta)$ for all $\eta \in\left[\eta_{\min }, \eta_{\max }\right]$, both parties adopt the same policy, i.e., $m^{L}=m^{R}$.

Lemma 1 specifies the equilibrium announcements of party $J, J=L, R$, which depend on whether $m^{J}$ is above or below $\hat{m}$. We now investigate the equilibrium relationship between $m^{J}$ and $\hat{m}$. Note that, as $\lambda^{I}$ approaches 1 , the value of $m^{J}$ that maximizes $V^{J}(m)$ approaches $m^{I}$. It follows that when the fraction of informed voters is large, $m^{J}$ falls on the same side of $\hat{m}$ as does $m^{I}$. That is, if if $m^{I}>\hat{m}$, then $m^{J}>\hat{m}$ and if $m^{I}<\hat{m}$, then $m^{J}<\hat{m}$. This also implies that, for $\lambda^{I}$ large enough, both parties choose policies on the same side of $\hat{m}$. In such circumstances, they also issue the same false report about the policy environment (either $\underline{\theta}$ or $\bar{\theta}$ ) and the same false report about the rival's policy position (either $\bar{m}$ or $\underline{m}$ ).

Next note that, as $\lambda^{I}$ approaches zero, the value of $m^{J}$ that maximizes $V^{J}(m)$ approaches

$$
m^{J}=\left\{\begin{array}{ll}
\underline{m} & \text { for } u(\underline{m}, \underline{\theta})-u(\bar{m}, \underline{\theta})>u(\bar{m}, \bar{\theta})-u(\underline{m}, \bar{\theta}) \\
\bar{m} & \text { for } u(\underline{m}, \underline{\theta})-u(\bar{m}, \underline{\theta})<u(\bar{m}, \bar{\theta})-u(\underline{m}, \bar{\theta})
\end{array} .\right.
$$

Evidently, in this case too, both parties adopt policies on the same side of $\hat{m}$ and they broadcast the same reports about the policy environment and about the rival party's position. It follows that as long as $u(\underline{m}, \underline{\theta})-u(\bar{m}, \underline{\theta})>u(\bar{m}, \bar{\theta})-u(\underline{m}, \bar{\theta})$ and $m^{I}>\hat{m}$ or $u(\underline{m}, \underline{\theta})-u(\bar{m}, \underline{\theta})<$ $u(\bar{m}, \bar{\theta})-u(\underline{m}, \bar{\theta})$ and $m^{I}<\hat{m}$, the optimal policy for party $J, m^{J}$, jumps from one side of $\hat{m}$ to the other side of $\hat{m}$ as $\lambda^{I}$ increases from zero to one. When $\pi(\eta) \neq 1-\pi(-\eta)$ for all $\eta \in\left[\eta_{\min }, \eta_{\max }\right]$, the policies of the two parties differ along this path and thus for some intermediate values of $\lambda^{I}$ one party chooses a policy below $\hat{m}$ while the other chooses one above $\hat{m}$. When this type of equilibrium occurs, the parties issue opposite extreme reports about the policy environment and opposite extreme reports about the policy position of the rival party; i.e., the reports polarize the electorate. This type of equilibrium is illustrated by the following example.

\section{Example}

Suppose $\underline{\theta}=1$ and $\bar{\theta}=2$. Let the utility function be given by

$$
u(m, \theta)=m-\frac{1}{2 \theta} m^{2} .
$$

These imply $\underline{m}=1$ and $\bar{m}=2$. In addition,

$$
\begin{aligned}
u(\underline{m}, \underline{\theta}) & =\frac{1}{2} ; u(\bar{m}, \underline{\theta})=0 \\
u(\bar{m}, \bar{\theta}) & =1 ; u(\underline{m}, \bar{\theta})=\frac{3}{4} \\
\hat{m} & =\sqrt{3} \simeq 1.73 .
\end{aligned}
$$


Now assume that $\lambda^{I}=0.4, \theta^{I}=(\sqrt{3}+2) / 2 \simeq 1.87$, and $\pi(\cdot)$ takes the form

$$
\pi(\eta)=\frac{e^{\eta}}{e^{\eta}+5}
$$

Using these properties and values, we obtain

$$
\begin{aligned}
& V^{L}(m)-\left(1-\lambda^{I}\right)\left[\ln \left(e^{\eta_{\min }}+5\right)-\eta_{\min }\right]= \\
& \lambda^{I}\left(m-\frac{1}{2 \theta^{I}} m^{2}\right)+\left(1-\lambda^{I}\right)\left\{\begin{array}{cl}
m-\frac{1}{2} m^{2}-\ln \left(e^{m-\frac{1}{2} m^{2}}+5\right) & \text { for } m \leq \hat{m}, \\
m-\frac{1}{4} m^{2}-\frac{3}{4}-\ln \left(e^{m-\frac{1}{4} m^{2}-\frac{3}{4}}+5\right) & \text { for } m \geq \hat{m},
\end{array}\right. \\
& V^{R}(m)=\lambda^{I}\left(m-\frac{1}{2 \theta^{I}} m^{2}\right)-\left(1-\lambda^{I}\right)\left\{\begin{array}{cl}
\ln \left(e^{-m+\frac{1}{2} m^{2}}+5\right) & \text { for } m \leq \hat{m}, \\
\ln \left(e^{\frac{3}{4}-m+\frac{1}{4} m^{2}-}+5\right) & \text { for } m \geq \hat{m} .
\end{array}\right.
\end{aligned}
$$

The first figure below plots $V^{R}(m)$, which takes the form

$$
\begin{aligned}
V^{R}(m)= & \max \left\{0.4\left(m-\frac{1}{\sqrt{3}+2} m^{2}\right)-(1-0.4) \ln \left(\exp \left(-\left(m-m^{2} / 2\right)\right)+5\right),\right. \\
& \left.0.4\left(m-\frac{1}{\sqrt{3}+2} m^{2}\right)-(1-0.4) \ln \left(\exp \left(3 / 4-\left(m-m^{2} / 4\right)\right)+5\right)\right\} .
\end{aligned}
$$

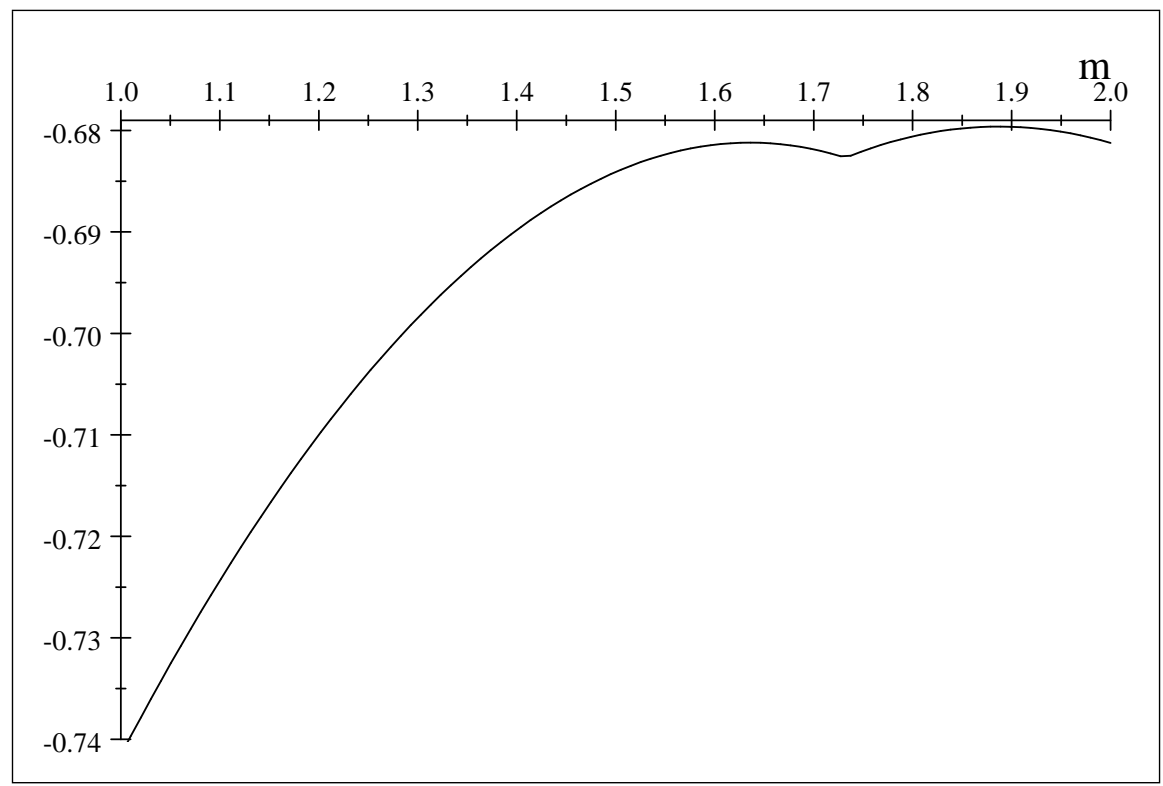

In this case the optimal strategy of $R$ is $m^{R} \approx 1.9>\hat{m}$. The next figure plots

$$
\begin{aligned}
& V^{L}(m)=2.7716 \\
& +\max \left\{0.4\left(m-\frac{1}{\sqrt{3}+2} m^{2}\right)+(1-0.4)\left(m-\frac{1}{2} m^{2}-\ln \left(\exp \left(m-\frac{1}{2} m^{2}\right)+5\right)\right)\right. \\
& \left.0.4\left(m-\frac{1}{\sqrt{3}+2} m^{2}\right)+(1-0.4)\left(m-\frac{1}{4} m^{2}-\frac{3}{4}-\ln \left(\exp \left(m-\frac{1}{4} m^{2}-\frac{3}{4}\right)+5\right)\right)\right\}
\end{aligned}
$$


where assuming $\eta_{\min }=-3$ yields $\left(1-\lambda^{I}\right)\left[\ln \left(e^{\eta_{\min }}+5\right)-\eta_{\min }\right] \simeq 2.7716$.

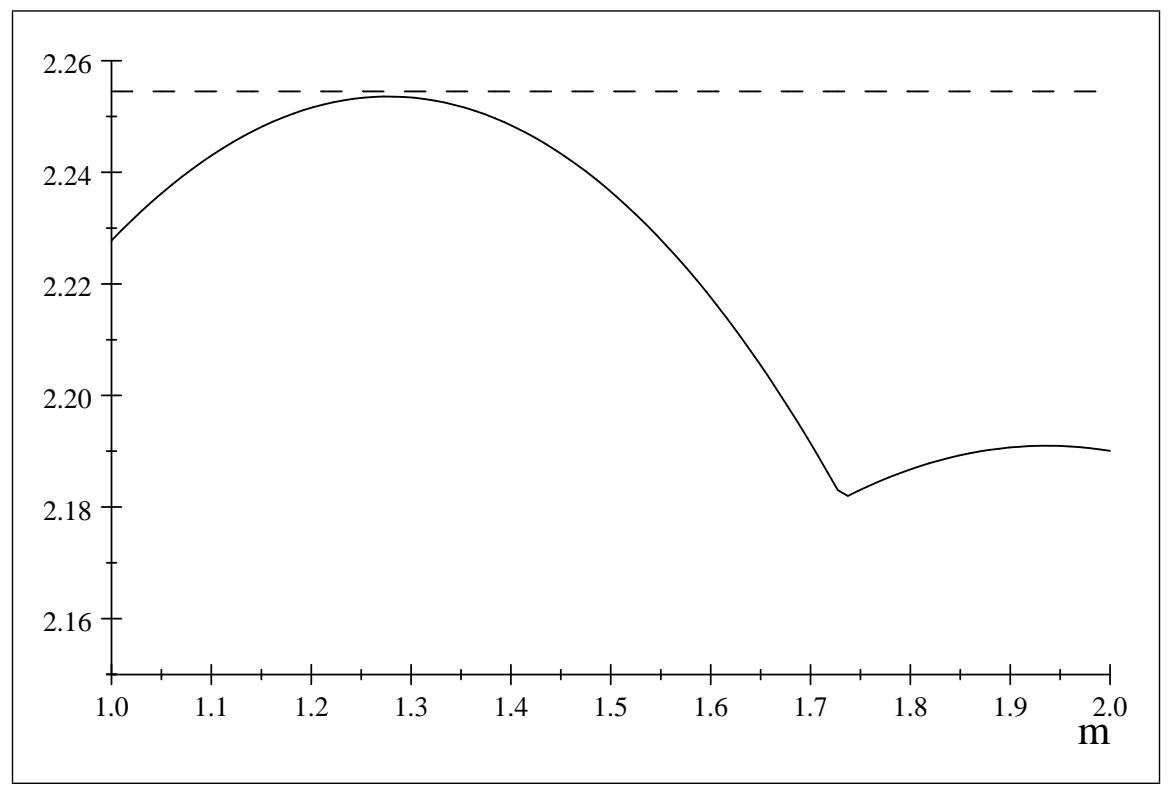

In this case, the optimal strategy of $L$ is $m^{L} \simeq 1.3<\hat{m}$. It follows that in equilibrium the two parties chooses policies on the opposite side of $\hat{m}$. The different incentives for the two parties are generated by the probability function $\pi(\eta)$. It satisfies

$$
\pi(\eta)+\pi(-\eta)=\frac{e^{\eta}}{e^{\eta}+5}+\frac{e^{-\eta}}{e^{-\eta}+5}=\frac{2+5\left(e^{\eta}+e^{-\eta}\right)}{26+5\left(e^{\eta}+e^{-\eta}\right)}<1
$$

and therefore $\pi(\eta)<1-\pi(-\eta)$ for all $\eta$. Thus, a voter with a given leaning $\eta$ toward party $R$ is less likely to hear news from a source biased toward party $R$ than is a voter of comparable leaning $-\eta$ toward party $L$ likely to hear news from a source biased toward party $L$.

\section{Appendix for Section 5}

In Section 5 of the paper, we discuss the case in which the media cannot misrepresent the policy positions adopted by the parties in the first stage of the game. We also assume that $F(\eta)=$ $\left(\eta-\eta_{\min }\right) /\left(\eta_{\max }-\eta_{\min }\right)$ is a uniform distribution on support $\left[\eta_{\min }, \eta_{\max }\right]$. As before, we assume that the support is broad enough so that a strictly positive fractions of voters favors each party no matter what are the beliefs about the pliable policy environment and the parties' positions. In this case, (5) can be expressed as

$$
\begin{aligned}
\left(\eta_{\max }-\eta_{\min }\right) s^{L} & =\lambda^{I}\left[u\left(m^{L}, \theta^{I}\right)-u\left(m^{R}, \theta^{I}\right)-\eta_{\min }\right] \\
& +\left(1-\lambda^{I}\right)\left\{\int_{\eta_{\min }}^{u\left(m^{L}, \theta^{L}\right)-u\left(m^{R}, \theta^{L}\right)}[1-\pi(\eta)] d \eta+\int_{\eta_{\min }}^{u\left(m^{L}, \theta^{R}\right)-u\left(m^{R}, \theta^{R}\right)} \pi(\eta) d \eta\right\} .
\end{aligned}
$$


From equation (2) in the main text,

$$
\theta^{J}=\left\{\begin{array}{ll}
\bar{\theta} & \text { for } m^{J}>m^{\widetilde{J}} \\
\underline{\theta} & \text { for } m^{J}<m^{\widetilde{J}}
\end{array} \quad, J=L, R .\right.
$$

Again, party $L$ seeks to maximize $s^{L}$ while party $R$ seeks to minimize it.

\section{Simultaneous Moves}

We first consider the game in which the parties move simultaneously in the first stage. First, we show that there exists no pure-strategy equilibrium with policy convergence. The argument proceeds as follows. Suppose that there exists an $\tilde{m} \in[\underline{m}, \bar{m}]$ such that $m^{L}=m^{R}=\tilde{m}$ is an equilibrium in the simultaneous-move game of the first stage. Then (10) implies $s^{L}=s_{0}^{L}$, where

$$
\left(\eta_{\max }-\eta_{\min }\right) s_{0}^{L}=-\lambda^{I} \eta_{\min }+\left(1-\lambda^{I}\right)\left\{\int_{\eta_{\min }}^{0}[1-\pi(\eta)] d \eta+\int_{\eta_{\min }}^{0} \pi(\eta) d \eta\right\} .
$$

Now consider a deviation by party $L$ to $m^{L}=\tilde{m}+\varepsilon, \varepsilon>0$ but small. If $\tilde{m}$ is the equilibrium strategy for party $L$ it has to be the case that $s_{0}^{L}$ is larger or equal to the vote share party $L$ would obtain under this deviation. Using (10)-(12), this implies

$$
\begin{aligned}
\left(1-\lambda^{I}\right)\left\{\int_{\eta_{\min }}^{0}[1-\pi(\eta)] d \eta+\int_{\eta_{\min }}^{0} \pi(\eta) d \eta\right\} \geq & \lambda^{I}\left[u\left(\tilde{m}+\varepsilon, \theta^{I}\right)-u\left(\tilde{m}, \theta^{I}\right)\right] \\
& +\left(1-\lambda^{I}\right) \int_{\eta_{\min }}^{u(\tilde{m}+\varepsilon, \bar{\theta})-u(\tilde{m}, \bar{\theta})}[1-\pi(\eta)] d \eta \\
& +\left(1-\lambda^{I}\right) \int_{\eta_{\min }}^{u(\tilde{m}+\varepsilon, \underline{\theta})-u(\tilde{m}, \underline{\theta})} \pi(\eta) d \eta .
\end{aligned}
$$

Using a first-order approximation to the right-hand side of this inequality, we have

$$
0 \geq \lambda^{I} u_{m}\left(\tilde{m}, \theta^{I}\right)+\left(1-\lambda^{I}\right)\left\{[1-\pi(0)] u_{m}(\tilde{m}, \bar{\theta})+\pi(0) u_{m}(\tilde{m}, \underline{\theta})\right\}
$$

A similar analysis for a downward deviation by party $L$, to $m^{L}=\tilde{m}-\varepsilon, \varepsilon>0$ but very small, implies

$$
0 \geq-\lambda^{I} u_{m}\left(\tilde{m}, \theta^{I}\right)-\left(1-\lambda^{I}\right)\left\{[1-\pi(0)] u_{m}(\tilde{m}, \underline{\theta})+\pi(0) u_{m}(\tilde{m}, \bar{\theta})\right\} .
$$

Likewise, a similar analysis of small deviations available to party $R$, which seeks to minimize $s^{L}$, implies that

$$
0 \leq-\lambda^{I} u_{m}\left(\tilde{m}, \theta^{I}\right)-\left(1-\lambda^{I}\right)\left\{[1-\pi(0)] u_{m}(\tilde{m}, \underline{\theta})+\pi(0) u_{m}(\tilde{m}, \bar{\theta})\right\}
$$

and

$$
0 \leq \lambda^{I} u_{m}\left(\tilde{m}, \theta^{I}\right)+\left(1-\lambda^{I}\right)\left\{[1-\pi(0)] u_{m}(\tilde{m}, \bar{\theta})+\pi(0) u_{m}(\tilde{m}, \underline{\theta})\right\} .
$$


The four inequalities together imply

$$
0=\lambda^{I} u_{m}\left(\tilde{m}, \theta^{I}\right)+\left(1-\lambda^{I}\right)\left\{[1-\pi(0)] u_{m}(\tilde{m}, \underline{\theta})+\pi(0) u_{m}(\tilde{m}, \bar{\theta})\right\}
$$

and

$$
0=\lambda^{I} u_{m}\left(\tilde{m}, \theta^{I}\right)+\left(1-\lambda^{I}\right)\left\{[1-\pi(0)] u_{m}(\tilde{m}, \bar{\theta})+\pi(0) u_{m}(\tilde{m}, \underline{\theta})\right\}
$$

and thus

$$
\left(1-\lambda^{I}\right)[1-2 \pi(0)]\left[u_{m}(\tilde{m}, \bar{\theta})-u_{m}(\tilde{m}, \underline{\theta})\right]=0 .
$$

Supermodularity implies $u_{m}(\tilde{m}, \bar{\theta})>u_{m}(\tilde{m}, \underline{\theta})$. It follows that, for $\lambda^{I}<1$, this condition is satisfied if and only if $\pi(0)=1 / 2$. We therefore conclude that whenever some individuals are ill-informed and $\pi(0) \neq 1 / 2$, there exists no equilibrium with policy convergence.

We next examine the conditions for a pure strategy equilibrium with divergent policies. Without loss of generality, we consider the case in which $m^{L}>m^{R}$. In this case $\theta^{L}=\bar{\theta}$ and $\theta^{R}=\underline{\theta}$ and therefore

$$
\begin{aligned}
\left(\eta_{\max }-\eta_{\min }\right) s^{L} & =\lambda^{I}\left[u\left(m^{L}, \theta^{I}\right)-u\left(m^{R}, \theta^{I}\right)-\eta_{\min }\right] \\
& +\left(1-\lambda^{I}\right)\left\{\int_{\eta_{\min }}^{u\left(m^{L}, \bar{\theta}\right)-u\left(m^{R}, \bar{\theta}\right)}[1-\pi(\eta)] d \eta+\int_{\eta_{\min }}^{u\left(m^{L}, \underline{\theta}\right)-u\left(m^{R}, \underline{\theta}\right)} \pi(\eta) d \eta\right\} .
\end{aligned}
$$

Since $L$ maximizes $s^{L}$, its choice of policy must satisfy

$$
\begin{array}{r}
\lambda^{I} u_{m}\left(m^{L}, \theta^{I}\right) \\
+\left(1-\lambda^{I}\right)\left\{1-\pi\left[u\left(m^{L}, \bar{\theta}\right)-u\left(m^{R}, \bar{\theta}\right)\right]\right\} u_{m}\left(m^{L}, \bar{\theta}\right) \\
+\left(1-\lambda^{I}\right) \pi\left[u\left(m^{L}, \underline{\theta}\right)-u\left(m^{R}, \underline{\theta}\right)\right] u_{m}\left(m^{L}, \underline{\theta}\right)=0 .
\end{array}
$$

Also, since $R$ minimizes $s^{L}$, its choice of policy must satisfy

$$
\begin{array}{r}
-\lambda^{I} u_{m}\left(m^{R}, \theta^{I}\right) \\
-\left(1-\lambda^{I}\right)\left\{1-\pi\left[u\left(m^{L}, \bar{\theta}\right)-u\left(m^{R}, \bar{\theta}\right)\right]\right\} u_{m}\left(m^{R}, \bar{\theta}\right) \\
-\left(1-\lambda^{I}\right) \pi\left[u\left(m^{L}, \underline{\theta}\right)-u\left(m^{R}, \underline{\theta}\right)\right] u_{m}\left(m^{R}, \underline{\theta}\right)=0 .
\end{array}
$$

Moreover, since each party can mimic the policy of its rival, the resulting vote share $s^{L}$ cannot be larger than nor smaller than the share that obtains when $m^{L}=m^{R}$. Using (13), this implies

$$
\begin{aligned}
\left(1-\lambda^{I}\right)\left\{\int_{\eta_{\min }}^{0}[1\right. & \left.-\pi(\eta)] d \eta+\int_{\eta_{\min }}^{0} \pi(\eta) d \eta\right\}=\lambda^{I}\left[u\left(m^{L}, \theta^{I}\right)-u\left(m^{R}, \theta^{I}\right)\right] \\
& +\left(1-\lambda^{I}\right)\left\{\int_{\eta_{\min }}^{u\left(m^{L}, \bar{\theta}\right)-u\left(m^{R}, \bar{\theta}\right)}[1-\pi(\eta)] d \eta+\int_{\eta_{\min }}^{u\left(m^{L}, \underline{\theta}\right)-u\left(m^{R}, \underline{\theta}\right)} \pi(\eta) d \eta\right\} .
\end{aligned}
$$


The last three equations comprise an overdetermined system that would need to be satisfied by the two variables, $m^{L}$ and $m^{R}$, such that satisfy $m^{L}>m^{R}$. Generically, no such solution exists. We therefore conclude:

Lemma 4 Let the policy choices $m^{J}, J=L, R$, be known to all voters, let the fraction of informed voters be smaller than one, i.e., $\lambda^{I}<1$, and let $\pi(0) \neq 1 / 2$. Then, if both parties move simultaneously in the first stage of the game, there exists (generically) no equilibrium in pure strategies

We have not been able to derive interesting insights about the properties of mixed-strategy equilibria for this game.

\section{Sequential Moves}

We now consider the game in which an incumbent, say party $R$, moves before the challenger when choosing its position in the initial stage of the game. To characterize the best response for the challenger, party $L$, observe that it follows from (10)-(11) that party $L$ obtains the share $s_{\text {above }}^{L}\left(m^{L}, m^{R}\right)$ of the votes if it chooses $m^{L}>m^{R}$ and the share $s_{\text {below }}^{L}\left(m^{L}, m^{R}\right)$ if it chooses $m^{L}<m^{R}$, where

$$
\begin{aligned}
\left(\eta_{\max }-\eta_{\min }\right) s_{\text {above }}^{L}\left(m^{L}, m^{R}\right) & \equiv \lambda^{I}\left[u\left(m^{L}, \theta^{I}\right)-u\left(m^{R}, \theta^{I}\right)-\eta_{\min }\right] \\
& +\left(1-\lambda^{I}\right)\left\{\int_{\eta_{\min }}^{u\left(m^{L}, \bar{\theta}\right)-u\left(m^{R}, \bar{\theta}\right)}[1-\pi(\eta)] d \eta+\int_{\eta_{\min }}^{u\left(m^{L}, \underline{\theta}\right)-u\left(m^{R}, \underline{\theta}\right)} \pi(\eta) d \eta\right\} \\
\left(\eta_{\max }-\eta_{\min }\right) s_{\text {below }}^{L}\left(m^{L}, m^{R}\right) & \equiv \lambda^{I}\left[u\left(m^{L}, \theta^{I}\right)-u\left(m^{R}, \theta^{I}\right)-\eta_{\min }\right] \\
& +\left(1-\lambda^{I}\right)\left\{\int_{\eta_{\min }}^{u\left(m^{L}, \underline{\theta}\right)-u\left(m^{R}, \underline{\theta}\right)}[1-\pi(\eta)] d \eta+\int_{\eta_{\min }}^{u\left(m^{L}, \bar{\theta}\right)-u\left(m^{R}, \bar{\theta}\right)} \pi(\eta) d \eta\right\} .
\end{aligned}
$$

The largest vote share that party $L$ can secure in response to $m^{R}$ is

$$
s_{\max }^{L}\left(m^{R}\right)=\max \left\{\max _{m \in\left[\underline{m}, m^{R}\right]} s_{\text {below }}^{L}\left(m, m^{R}\right), \max _{m \in\left[m^{R}, \bar{m}\right]} s_{\text {above }}^{L}\left(m, m^{R}\right)\right\} .
$$

Under these conditions, the equilibrium strategy of party $R$ is

$$
m^{R}=\arg \min _{m \in[\underline{m}, \bar{m}]} s_{\max }^{L}\left(m^{R}\right) .
$$

In this sequential game, the existence of an equilibrium in pure strategies is assured.

Next, note that party $L$ always has the option to choose $m^{L}=m^{R}$, in which case its vote share would be $s_{0}^{L}$, defined in (12). For this reason, the equilibrium vote share satisfies $s^{L} \geq s_{0}^{L}$ and if there exists an $m^{R}$ such that $s_{\max }^{L}\left(m^{R}\right)=s_{0}^{L}$, this $m^{R}$ must be an equilibrium play for party $R$. 
We now show that there exists no equilibrium with policy convergence when $\lambda^{I}<1$ and $\pi(0)<1 / 2$. The argument proceeds as follows. Suppose there were to exist an $\tilde{m}$ such that $m^{R}=\tilde{m} \in(\underline{m}, \bar{m})$ and $m^{L}=\tilde{m}$ are equilibrium plays for the incumbent and the challenger, respectively. Then for $\varepsilon>0$,

$$
\begin{aligned}
& s_{\text {above }}^{L}(\tilde{m}+\varepsilon, \tilde{m}) \leq s_{0}^{L}=s_{\text {above }}^{L}(\tilde{m}, \tilde{m}), \\
& s_{\text {below }}^{L}(\tilde{m}-\varepsilon, \tilde{m}) \leq s_{0}^{L}=s_{\text {above }}^{L}(\tilde{m}, \tilde{m}) .
\end{aligned}
$$

Using (14) and (15), these inequalities imply

$$
\begin{gathered}
0 \geq \lambda^{I} u_{m}\left(\tilde{m}, \theta^{I}\right)+\left(1-\lambda^{I}\right)\left\{[1-\pi(0)] u_{m}(\tilde{m}, \bar{\theta})+\pi(0) u_{m}(\tilde{m}, \underline{\theta})\right\}, \\
0 \geq-\lambda^{I} u_{m}\left(\tilde{m}, \theta^{I}\right)-\left(1-\lambda^{I}\right)\left\{[1-\pi(0)] u_{m}(\tilde{m}, \underline{\theta})+\pi(0) u_{m}(\tilde{m}, \bar{\theta})\right\} .
\end{gathered}
$$

Summing the two inequalities yields

$$
0 \geq\left(1-\lambda^{I}\right)[1-2 \pi(0)]\left[u_{m}(\tilde{m}, \bar{\theta})-u_{m}(\tilde{m}, \underline{\theta})\right]
$$

The supermodularity of $u(m, \theta)$ implies $u_{m}(\tilde{m}, \bar{\theta})>u_{m}(\tilde{m}, \underline{\theta})$. Therefore, this last inequality must be violated whenever $\lambda^{I}<1$ and $\pi(0)<1 / 2$. It is also easy to verify that neither $\tilde{m}=\underline{m}$ nor $\tilde{m}=\bar{m}$ is an equilibrium. For suppose that $\tilde{m}=\underline{m}$. Then only a deviation to $m^{L}=\underline{m}+\varepsilon$ is possible, in which case only (18) has to be satisfied, which becomes

$$
0 \geq \lambda^{I} u_{m}\left(\underline{m}, \theta^{I}\right)+\left(1-\lambda^{I}\right)\left\{[1-\pi(0)] u_{m}(\underline{m}, \bar{\theta})+\pi(0) u_{m}(\underline{m}, \underline{\theta})\right\} .
$$

But since $u_{m}\left(\underline{m}, \theta^{I}\right)>0, u_{m}(\underline{m}, \bar{\theta})>0$ and $u_{m}(\underline{m}, \underline{\theta})=0$, this inequality is violated. A similar argument establishes that $\tilde{m}=\bar{\theta}$ violates (19). We have proven

Lemma 5 Suppose the parties play sequentially in the first stage of the game and that their choices $m^{J}, J=L, R$, become known to all voters. Let the fraction of informed voters be smaller than one, i.e., $\lambda^{I}<1$, and let $\pi(0)<1 / 2$. Then, there does not exist an equilibrium with policy convergence; i.e. $m^{R} \neq m^{L}$ under these circumstances.

We now provide an example that has $\pi(\eta)>1-\pi(-\eta)$ for all $\eta \in\left[\eta_{\min }, \eta_{\max }\right]$, so that $\pi(0)>1 / 2$, in which there exists an equilibrium with $m^{L}=m^{R}$.

\section{Example}

Suppose $\underline{\theta}=1$ and $\bar{\theta}=2$. Let the utility function be given by

$$
u(m, \theta)=m-\frac{1}{2 \theta} m^{2}
$$


These imply $\underline{m}=1$ and $\bar{m}=2$. Moreover,

$$
\begin{aligned}
& u(\underline{m}, \underline{\theta})=\frac{1}{2} ; u(\bar{m}, \underline{\theta})=0 \\
& u(\bar{m}, \bar{\theta})=1 ; u(\underline{m}, \bar{\theta})=\frac{3}{4} .
\end{aligned}
$$

Now assume that $\lambda^{I}=0.4, \theta^{I}=(\sqrt{3}+2) / 2 \simeq 1.87$ and that the probability function takes the form

$$
\pi(\eta)=\frac{e^{\eta}}{e^{\eta}+0.5}
$$

This probability function implies $\pi(\eta)>1-\pi(-\eta)$ for all $\eta$ and therefore $\pi(0)>1 / 2$. Using these properties and values, we obtain

$$
\begin{aligned}
& \eta_{\min }+\left(\eta_{\max }-\eta_{\min }\right) s_{\text {above }}^{L}\left(m, m^{R}\right) \\
& \equiv 0.4\left\{m-\frac{1}{(\sqrt{3}+2)} m^{2}-\left[m^{R}-\frac{1}{(\sqrt{3}+2)}\left(m^{R}\right)^{2}\right]\right\} \\
& +0.6 \ln \left(e^{m-\frac{1}{2} m^{2}-\left[m-\frac{1}{2}\left(m^{R}\right)^{2}\right]}+0.5\right) \\
& +0.6\left\{\left[m-\frac{1}{4} m^{2}-\left[m^{R}-\frac{1}{4}\left(m^{R}\right)^{2}\right]-\ln \left(e^{m-\frac{1}{4} m^{2}-\left[m^{R}-\frac{1}{4}\left(m^{R}\right)^{2}\right]}+0.5\right)\right]\right\}, \\
& \eta_{\min }+\left(\eta_{\max }-\eta_{\min }\right) s_{\text {below }}^{L}\left(m, m^{R}\right) \\
& \equiv 0.4\left\{m-\frac{1}{(\sqrt{3}+2)} m^{2}-\left[m^{R}-\frac{1}{(\sqrt{3}+2)}\left(m^{R}\right)^{2}\right]\right\} \\
& +0.6 \ln \left(e^{m-\frac{1}{4} m^{2}-\left[m^{R}-\frac{1}{4}\left(m^{R}\right)^{2}\right]}+0.5\right) \\
& +0.6\left\{\left[m-\frac{1}{2} m^{2}-\left[m^{R}-\frac{1}{2}\left(m^{R}\right)^{2}\right]-\ln \left(e^{m-\frac{1}{2} m^{2}-\left[m^{R}-\frac{1}{2}\left(m^{R}\right)^{2}\right]}+0.5\right)\right]\right\},
\end{aligned}
$$

In Figure 2, reproduced below, the dashed curve plots $\eta_{\min }+\left(\eta_{\max }-\eta_{\min }\right) s_{\text {below }}^{L}$ while the the solid curve plots $\eta_{\min }+\left(\eta_{\max }-\eta_{\min }\right) s_{\text {above }}^{L}$ for $m^{R}=1.5$. As is clear from the figure, $m^{L}=m^{R}$ is the best response of party $L$ to $m^{R}=1.5$. That is, the example has an equilibrium with policy convergence. 


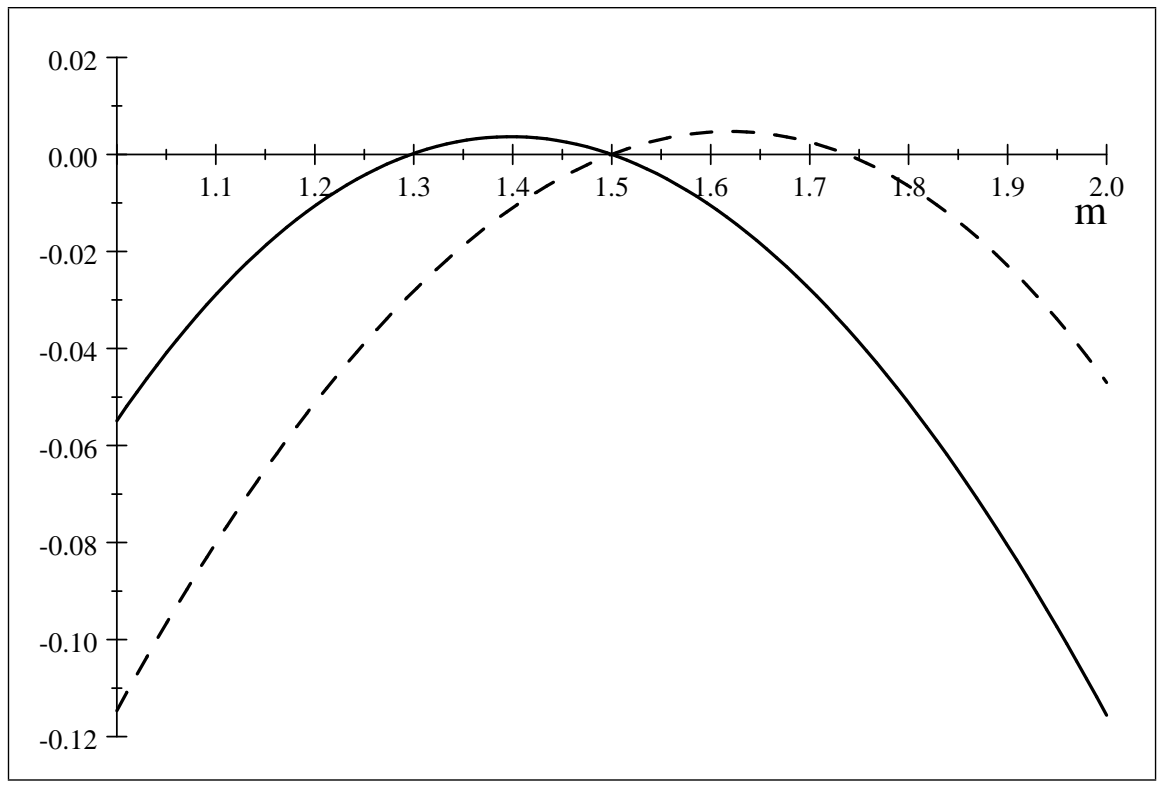

